\title{
Construction and Immunogenicity of a Recombinant Pseudorabies Virus Expressing the Rabies Virus Glycoprotein and EGFP
}

\author{
${ }^{1,2}$ Naijun Han, ${ }^{1,2}$ Yewei Li, ${ }^{1,2}$ Chenglong Sun, ${ }^{3}$ Qingguo Meng, \\ ${ }^{4}$ Feifei Song, ${ }^{1,2}$ Yang Yang, ${ }^{2}$ Shoufeng Zhang and ${ }^{2}$ Rongliang Hu \\ ${ }^{1}$ College of Animal Science and Veterinary Medicine, \\ Jilin University, 5333 Xi' an Road, 130062 Changchun, China \\ ${ }^{2}$ Academy of Military Medical Sciences, Institute of Veterinary Research, \\ 666 Liuyingxilu Road, 130122 Changchun, China \\ ${ }^{3}$ Jilin Entry-Exit Inspection and Quarantine Bureau, \\ 1301 Puyang Street, 130062 Changchun, China \\ ${ }^{4}$ Qingdao Agricultural University, 700 Changcheng Road, \\ Chengyang, 266109 Qingdao, China
}

\begin{abstract}
The rabies virus glycoprotein is an efficient vaccine antigen particularly for the oral vaccine of wildlife. Pseudorabies virus contains many non-essential genes and was usually used to construct recombinant vaccines as a viral vector to deliver foreign genes into the target animals. In this study, a recombinant pseudorabies virus rPRV/SRV9G/EGFP, expressing EGFP and rabies virus (SRV9 strain) glycoprotein was constructed by homologous recombination in the $U L 21$ gene. The immunogenicity was evaluated as a vaccine in mice. In this trial, thirty mice were administered with the recombinant vaccine orally. This recombinant virus was demonstrated to be safe for mice by oral administration and could induce neutralizing antibody level above $0.5 \mathrm{IU} \mathrm{mL} \mathrm{m}^{-1}$ at 3 weeks after the vaccination. The results suggest that rPRV/SRV9G/EGFP was an effective oral vaccine candidate against rabies and $U L 21$ gene could be a target insertion area for foreign genes.
\end{abstract}

Key words: Rabies Virus (RV), Pseudorabies Virus (PRV), UL21 gene, SRV9 glycoprotein, oral vaccine, homologous recombination

\section{INTRODUCTION}

Rabies is a deadly disease that continues to menace all mammals (including humans) in many areas of the world especially Asia and Africa (Hu et al., 2006). Humans and animals are usually infected with the rabies virus through a bite or a scratch by a rabid animal. In Africa and Asia, the vast majority of human rabies is associated with virus transmission from dogs although, wild carnivores and bats are also important reservoirs (WHO, 2005). Cell culture vaccines and anti-rabies virus immunoglobulins cost too much while oral vaccine is cheap and convenient to control the dog and wildlife rabies. So, oral vaccination is an important part of wildlife rabies control programs. A number of live vaccines such as some attenuated rabies virus strains (ERA/SAD/SAG) and recombinant vaccinia (V-RG) viruses have been used successfully in oral vaccination field programs to limit the spread of rabies in coyotes, foxes and raccoons in North America and Western Europe (Kieny et al., 1990; Brochier et al., 1990; Cliquet and Barrat, 2008).
The rabies virus genome produces five monocistronic mRNAs encoding Nucleoprotein $(\mathrm{N})$, Phosphoprotein $(\mathrm{P})$, Matrix protein (M), Glycoprotein (G) and viral RNA-dependent RNA polymerase (L), respectively (Wunner and Larson, 1988). The G protein is a $65 \mathrm{kDa}$ type I transmembrane protein consisting of an extracellular domain, a transmembrane domain and a cytoplasmic domain. It is the only protein on the surface of mature virus and plays an important role in virus infection and also a major antigenic stimulus of the host immune system inducing neutralizing antibodies (Cox and Dietzschold, 1977).

Pseudorabies Virus (PRV), a member of herpesvirus type I has a broad host range including sheep, cattle, dogs and rodents (Klupp et al., 2004). PRV contains many non-essential genes such as Thymidine Kinase (TK), Protein Kinase (PK), Glycoprotein E (gE), glycoprotein I (gI) and glycoprotein $\mathrm{G}(\mathrm{gG})$ which make insertion of foreign genes feasible (Klupp et al., 2004; Olsen et al., 2006). The PRV Bartha K-61 strain, used in the trial has

Corresponding Author: Rongliang Hu, Academy of Military Medical Sciences, Institute of Veterinary Research, 666 Liuyingxilu Road, 130122 Changchun, China 
been used as a live vaccine against Aujeszky's disease in swine for decades and has a good safety record. Because of so many advantages described before, the PRV was usually used to construct recombinant vaccines as a viral vector to deliver foreign genes into the target animals (Liu et al., 2008; Yuan et al., 2008). The UL21 protein of PRV is a capsid-associated protein involved in capsid maturation during cleavage and encapsidation of the viral genome and deletion of the pUL21 could result in a drastic decrease in the incorporation of the pUL 46, pUL49 and pUS3 tegument components into mature virions (De Wind et al., 1992; Wagenaar et al., 2001; Michael et al., 2007). The PRV Bartha K-61 strain has 3 amino acid mutations in UL21 protein that affect the virus virulence and ability to spread through neural circuits (Klupp et al., 1995). The virus lacking UL21 gene could be propagated on non-complementing cells and nasal mucosa explants indicating that UL21 protein are not required for viral replication in cell culture (Wagenaar et al., 2001; Klupp et al., 2005) and could be replaced by exogenous proteins.

In this study, the researchers generated a recombinant pseudorabies virus replaced the UL21 gene by a double expression cassette of EGFP and rabies virus SRV9 glycoprotein and its ability to induce protective immunity against virulent challenge was tested on BALB/c Mouse Model.

\section{MATERIALS AND METHODS}

Cells and virus: BHK-21 cells were grown in Dulecco's Modified Eagle's Medium (DMEM, Gibco BRL Life Technology Inc) supplemented with 100 IU penicillin $\mathrm{mL}^{-1}, 100 \mathrm{mg}$ streptomycin $\mathrm{mL}^{-1}$ and $3 \%(\mathrm{v} / \mathrm{v}$ ) Fetal Bovine Serum (FBS, Gibco) at $37^{\circ} \mathrm{C}$ in $5 \% \mathrm{CO}_{2}$. PRV vaccine strain Bartha-K61 was maintained in the laboratory, propagated and titrated in BHK- 21 cells. The pEGFP-N1 Vector was purchased from CLONTECH Laboratories, Inc. The G gene of RV strain SRV9 was amplified and cloned into $\mathrm{pMD} 18$-T vector as described before (Hu et al., 2006).

Construction of transfer plasmids: To construct the SRV9 glycoprotein expressing cassette, the SRV $G$ gene was cut from pMD18-T-SRV9G and inserted into the pcDNA3.1(+) vector and the name of the new plasmid was termed pcDNA-SRV9G. To produce the double expression cassette, the EGFP expressing cassette was cut from pEGFP-N1 vector and replaced the neomycin resistance gene of the pcDNA-SRV9G. The genomic DNA of PRV Bartha-K61 strain was extracted and purified as described previously (Jestin et al., 1990). The two fragments of the UL21 gene (GenBank accession NC_006151) were obtained by Polymerase Chain Reaction (PCR) amplification from PRV Bartha K-61 strain genomic DNA
Table 1: Primers used to amplify the homologous recombinant arms flanked

\begin{tabular}{ll}
\multicolumn{2}{c}{ UL21 gene } \\
\hline Primers & Primer sequence $^{\mathrm{a}}\left(5^{\prime}-3^{\prime}\right)$ \\
\hline F1 & TTGAGTACCAGAGCACGAT \\
R1 & ATGCACTCGACGAGGTAG \\
F2 & ATGCGGATGCTCGTGAAC \\
R2 & TATTGAGGACGATGGAGATGT \\
\hline
\end{tabular}

as the homologous recombinant arms with two pairs of specific primers as Table 1 . Then, to construction the transfer plasmid, the double expression cassette, flanked by the two fragments of PRV UL21 gene was cut from the pcDNA3.1-SRV9G-EGFP and inserted into the pPoly2 vector.

Generation of the recombinant rPRV/SRV9G/EGFP virus: The recombinantrPRV/SRV9G/EGFP was generated by co-transfection with the genomic DNA of PRV Bartha $\mathrm{K}-61$ and plasmid pUL21-SRV9G-EGFP via homologous recombination using FuGENE ${ }^{\circledR} \mathrm{HD}$ transfection reagent, according to manufacturer's instructions. At 3 days post transfection, virus-containing supernatant was collected and subcultured into BHK-21 cells for plaque purification. After $2 \mathrm{~h}$ incubation with recombinant virus containing supernatant, cell monolayers were overlaid with $2 \%$ low gelling temperature nutrient agar containing $10 \%(\mathrm{v} / \mathrm{v})$ heat-inactivated Fetal Bovine Serum (FBS) (Invitrogen Corporation, San Diego, CA, USA), $4 \mathrm{mmol} \mathrm{L}{ }^{-1} \mathrm{~L}-$ glutamine, $1.97 \mathrm{~g} \mathrm{~L}^{-1} \mathrm{NaHCO}_{3}$ for plaque isolation. Potential positive recombinants were screened by their green-fluorescing plaque phenotype under a fluorescence microscope. The green-fluorescing cell foci was picked by aspiration and seeded on BHK-21 cells. After six rounds of screening, plaques showing green fluorescence by fluorescent microscopy were harvested. Presence of SRV9 glycoprotein was detected by PCR amplification using specific primers for SRV9 G gene. Fg: 5'-CCGGAATTCCC ACCATGGTTCCTCAGGCTCTCC-3',Rg:5'-CGCGGATCC TTACAGTCTGGTCTCACCC-3'.

Western blot analysis of the glycoprotein expressed in BHK-21 cells: Recombinant virus rPRV/SRV9G/EGFP and PRV Bartha-K61 were grown in BHK-21 cells for $72 \mathrm{~h}$. About $20 \mu \mathrm{L}$ cell lysates were subjected to SDS Polyacrylamide Gel Electrophoresis (SDS-PAGE) followed by immunoblotting onto nitrocellulose membranes as described by Sui et al. (2010). Nonspecific antibodybinding sites were blocked with 5\% Dried Skim Milk (DSM) in Phosphate-Buffered Saline (PBS). Mouse anti-rabies virus glycoprotein monoclonal antibody and Horseradish Peroxidase (HRP)-conjugated goat anti-mouse IgG (SIGMA, St.Louis, MO, USA) were respectively used as the primary and secondary antibody at dilutions specified by the manufacturer. 3 , 3'-Diaminobenzidine tetrahydrochloride (DAB) was the substrate. 
Oral vaccination of rPRV/SRV9G/EGFP on mice: Ninety healthy 4 weeks old mice with no history of vaccination against pseudorabies or rabies were purchased from Animal Center of Institute of Changchun Biological Products, Changchun, China. They were randomly divided into three groups of 30 each. All mice were inoculated by oral route. Group 1 and 2 were respectively inoculated once with $1 \mathrm{~mL}$ of medium containing $6 \times 10^{6}$ Plaque Forming Units (PFU) of rPRV/SRV9G/EGFP or PRV. Group 3 was served as a negative control and inoculated with $1 \mathrm{~mL}$ of medium without any virus following the same administration. After immunization, serum samples were collected from the tail vein at various time-points for serological tests.

Neutralizing antibodies against rabies viruses: Mice sera were inactivated at $56^{\circ} \mathrm{C}$ for $30 \mathrm{~min}$. Rabies virus neutralizing antibody was determined by the Fluorescent Antibody Virus Neutralization (FAVN) test as described previously (Hu et al., 2006).

Data analysis: Data were analyzed by one-way ANOVA using the SPSS 13.0 Software package (SPSS Inc., Chicago, $\amalg, \mathrm{USA}$ ).

\section{RESULTS AND DISCUSSION}

Construction and identification of recombinant pseudorabies virus: After 6 cycles plaque isolation, almost all the cell foci showed green fluorescence under the fluorescence microscope (Fig. 1). In BHK-21 cells, the growth assay showed that there were no significant differences in plaque size and titers between the

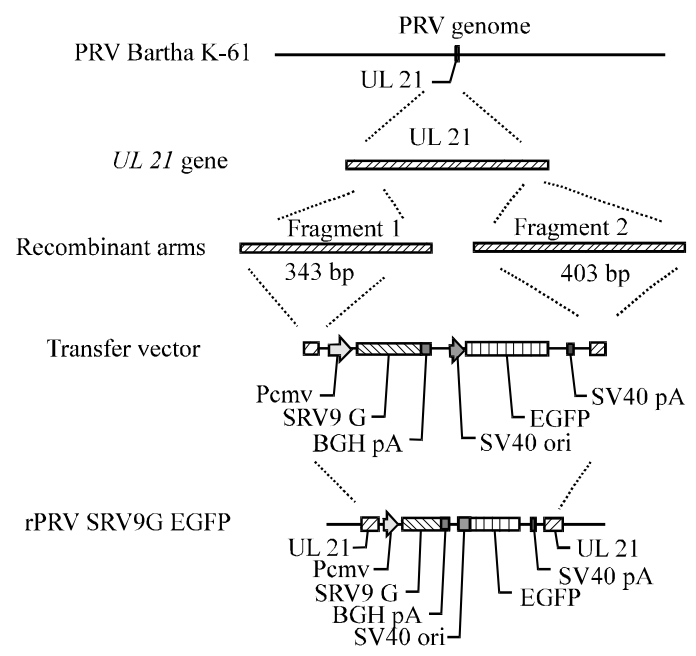

Fig. 1: Schematic diagram of the generation of recombinant $\mathrm{PRV}$ recombinant and parental virus. It indicated that the insertion of foreign genes in the UL21 gene does not affect the replication of PRV.

Rabies virus glycoprotein expression of rPRV/SRV9G/EGFP in BHK cells: The SRV 9 glycoprotein gene was homologous recombined into the PRV genome that was verified by PCR with specific primers for $S R V 9 G$ gene. The Western blot results also demonstrated correct expression of SRV9 glycoprotein $(65 \mathrm{kDa})$ in the identified recombinant virus (Fig. 2).

Neutralizing antibody against rabies: Neutralizing antibody levels against rabies virus of group 1 mice were $>0.5 \mathrm{IU} \mathrm{mL}^{-1}$ from the $3 \mathrm{rd}$ week onward and lasted for $>3$ months keeping this level. Data represent the mean value with standard deviation (Fig. 3).

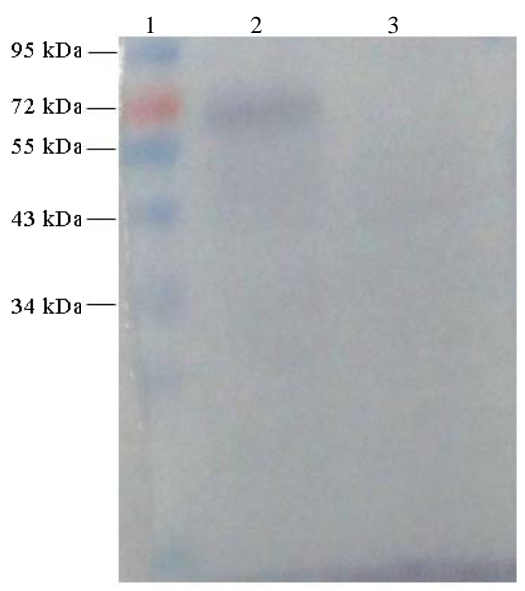

Fig. 2: Western blot analysis of SRV9 glycoprotein expressed by rPRV/SRV9G/EGFP. Lane 1: PageRuler ${ }^{\mathrm{TM}}$ Prestained Protein Ladder (NEB Inc, England), Lane 2: BHK cell infected with rPRV/SRV9G/EGFP; Lane 3: BHK cell infected with Bartha K-61

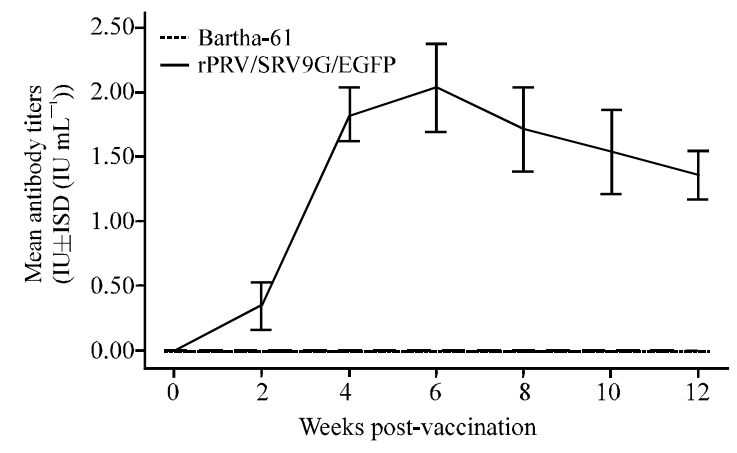

Fig. 3: Neutralizing antibody levels induced by rPRV/ SRV9G/EGFP in mice 
The development of oral vaccines to control rabies in China and other developing countries is necessary and urgent. In the recent years, there are several types of live vectors used in construction of rabies recombinant vaccines. In the North America and Western Europe, the Vaccinia virus-based rabies vaccine has been used to control wildlife rabies. However, in the immunodeficient people, contact with the Vaccinia virus-based rabies vaccine could cause adverse reactions (Brochier et al., 1990). Human adenovirus type 5 used for rabies vaccine development also has safety problems in human (Yarosh et al., 1996). Pseudorabies virus has long been explored for vaccine development (Qian and $\mathrm{Li}, 2004$; Hong et al., 2007). The PRV strain Bartha $\mathrm{K}-61$ is an approved vaccine strain for prevention of porcine pseudorabies in China. It should be safe for all domestic species as well as pigs. The researchers constructed a recombinant pseudorabies expressing fusion of the ectodomain of the rabies virus glycoprotein with EGFP which could effectively induce the rabies virus neutralizing antibody in dogs (Yuan et al., 2008). However, compared to the commercial inactivated rabies vaccine, the neutralizing antibody level is much lower. The relatively low response was probably related to the ectodomain of the glycoprotein and the fusional expression. So, in this study, the resaerchers amplified the whole glycoprotein of SRV9 and constructed a double expression cassette individually expressing rabies virus glycoprotein and EGFP. It induced higher neutralizing antibody level than that of the fusional expression recombinant pseudorabies and provided full protection against rabies virus challenge. The results indicated that the transmembrane region of rabies virus glycoprotein should be necessary in exhibiting antigens of the recombinant virus. Neutralizing antibody was monitored for 3 months at the end of which VNA titers were still at a high level above $0.5 \mathrm{IU} \mathrm{mL}^{-1}$

UL21 locus of PRV Bartha K-61 contains seven point mutations that induced 3 amino acid mutations of UL21 protein (Klupp et al., 1995). These mutations result in inefficient tegument assembly in PRV Bartha K-61 (Michael et al., 2007) and such a defect may diminish the infectivity of transmitted virions. In this study, researchers amplified two fragments of $U L 21$ gene used as a pair of homologous recombination arms. By homologous recombination, we inserted a double expression cassette of SRV9 glycoprotein and EGFP into the PRV Bartha K-61 genomic DNA and obtained a recombinant virus (Fig. 4). For a recombinant virus vaccine, it is important to verify that the insertion of foreign genes in the parental virus genome and the expression of foreign protein could not adversely affect

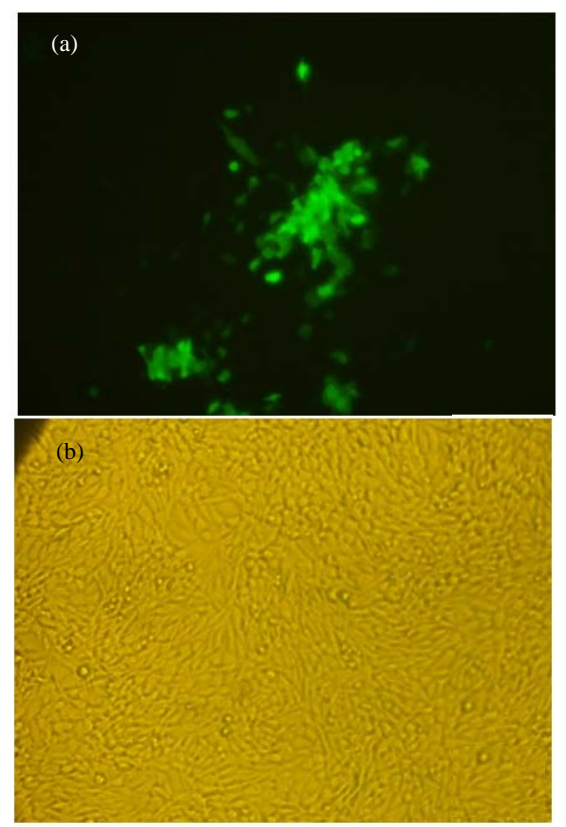

Fig. 4: Expression of EGFP in BHK cell foci by fluorescence microscopy; a) rPRV/SRV9G/EGFPinfected cells $36 \mathrm{~h}$ post-infection and b) Normal control cells

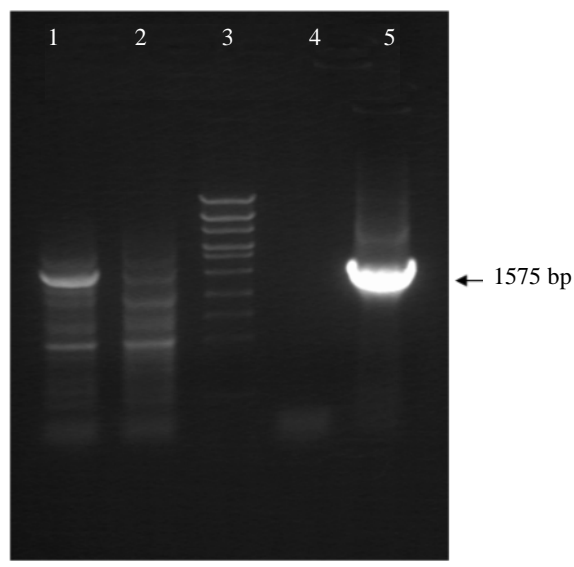

Fig. 5: SRV9 glycoprotein gene in the recombinant rPRV/SRV9G/EGFP was detected by PCR amplification; Lane 1: rPRV/SRV9G/EGFP; Lane 2: PRV; Lane 3: 6280 marker; Lane 4: Negative control; Lane 5: Positive control

viral growth. The growth assay showed that the recombinant viruses replicated in cells culture as efficiently as PRV vaccine strain Bartha K-61 and the final virus yields were very similar. The $U L$ gene has not been reported in development of recombinant PRV vaccines. The results showed that it was feasible to manipulate this region for insertion of foreign gene (s) (Fig. 5). 


\section{CONCLUSION}

The data in this trial shows that rPRV/SRV9G/EGFP provides long term, safe and economical immunization in mice. The research also suggests that Bartha K-61 might be used as vector to develop new types of oral vaccines and UL21 gene could be a target insertion area for foreign genes.

\section{ACKNOWLEDGEMENT}

This research was supported by grants from National 863 projects (High Technology Research and Development Program of China, Approval Nos. 2007AA02Z401, 2006AA02Z456 and 2006AA10A204).

\section{REFERENCES}

Brochier, B., I. Thomas, B. Bauduin, T. Leveau and P.P. Pastoret et al., 1990. Use of a vaccinia-rabies recombinant virus for the oral vaccination of foxes against rabies. Vaccine, 8: 101-104.

Cliquet, F. and J. Barrat, 2008. Efficacy and bait acceptance of vaccinia vectored rabies glycoprotein vaccine in captive foxes (Vulpes vulpes), raccoon dogs (Nyctereutes procyonoides) and $\operatorname{dogs}$ (Canis familiaris). Vaccine, 26: 4627-4638.

Cox, J.H. and B. Dietzschold, 1977. Rabies virus glycoprotein. II. Biological and serological characterization. Infect. Immunity, 16: 754-759.

De Wind, N., F. Wagenaar, J. Pol, T. Kimman and A. Berns, 1992. The pseudorabies virus homology of the herpes simplex virus UL21 gene product is a capsid protein which is involved in capsid maturation. J. Virol., 66: 7096-7103.

Hong, Q., P. Qian, X.M. Li, X.L. Yu and H.C. Chen, 2007. A recombinant pseudorabies virus co-expressing capsid proteins precursor P1-2A of FMDV and VP2 protein of porcine parvovirus: A trivalent vaccine candidate. Biotechnol. Lett., 29: 1677-1683.

Hu, R.L., S. Zhang, A.R. Fooks, H. Yuan and Y. Liu et al., 2006. Prevention of rabies virus infection in dogs by a recombinant canine adenovirus type- 2 encoding the rabies virus glycoprotein. Microb. Infect., 8: 1090-1097.

Jestin, A., T. Foulon, B. Pertuiset, P. Blanchard and M. Labourdet, 1990. Rapid detection of pseudorabies virus genomic sequences in biological samples from infected pigs using polymerase chain reaction DNA amplification. Vet. Microbiol., 23: 317-328.

Kieny, M.P., R. Lathe, R. Drillien, D. Spehner and S. Skory et al., 1990. Expression of rabies virus glycoprotein from a recombinant vaccinia virus. Nature, 312: 163-166.
Klupp, B.G., B. Lomniczi, N. Visser, W. Fuchs and T.C. Mettenleiter, 1995. Mutations affecting the UL21 gene contribute to avirulence of pseudorabies virus vaccine strain Bartha. Virol., 212: 466-473.

Klupp, B.G., C.J. Hengartner, T.C. Mettenleiter and L.W. Enquist, 2004. Complete, annotated sequence of the pseudorabies virus genome. J. Virol., 78: 424-440.

Klupp, B.G., S. Bottcher, H. Granzow, M. Kopp and T.C. Mettenleiter, 2005. Complex formation between the UL16 and UL21 tegument proteins of pseudorabies virus. J. Virol., 79: 1510-1522.

Liu, Q., S. Gao, L. Jiang, L. Shang and J. Men et al., 2008. A recombinant pseudorabies virus expressing TgSAG1 protects against challenge with the virulent Toxoplasma gondii $\mathrm{RH}$ strain and pseudorabies in $\mathrm{BALB} / \mathrm{c}$ mice. Microb. Infect., 10: 1355-1362.

Michael, K., B.G. Klupp, A. Karger and T.C. Mettenleiter, 2007. Efficient incorporation of tegument proteins pUL46, pUL49, and pUS3 into pseudorabies virus particles depends on the presence of pUL21. J. Virol., 81: 1048-1051.

Olsen, L.M., T.H. Ch'ng, J.P. Card and L.W. Enquist, 2006. Role of pseudorabies virus Us3 protein kinase during neuronal infection. J. Virol., 80: 6387-6398.

Qian, P. and X.M. Li, 2004. An approach to a FMD vaccine based on genetic engineered attenuated pseudorabies virus: One experiment using VPl gene alone generates an antibody responds on FMD and pseudorabies in swine. Vaccine, 22: 2129-2136.

Sui, X., J. Yin and X. Ren, 2010. Antiviral effect of diammonium glycyrrhizinate and lithium chloride on cell infection by pseudorabies herpesvirus. Antiviral Res., 85: 346-353.

WHO, 2005. WHO expert consultation on rabies. World Health Organization Technical Repot Series No. 931, pp: $1-88$.

Wagenaar, F., J.M. Pol, N. de Windb and T.G. Kimmanc, 2001. Deletion of the UL21 gene in Pseudorabies virus results in the formation of DNA-deprived capsids: An electron microscopy study. Vet. Res., 32: 47-54.

Wunner, W.H. and J.K. Larson, 1988. The molecular biology of rabies viruses. Rev. Infect. Dis., 4: $\mathrm{S} 771-7 \mathrm{~S} 84$.

Yarosh, O.K., A.I. Wandeler, F.L. Graham, J.B. Campbell and L. Prevec, 1996. Human adenovirus type 5 vectors expressing rabies glycoprotein. Vaccine, 14: $1257-1264$.

Yuan, Z., S. Zhang, Y. Liu, F. Zhang, A.R. Fooks, Q. Li and R. Hu, 2008. A recombinant pseudorabies virus expressing rabies virus glycoprotein: Safety and immunogenicity in dogs. Vaccine, 26: 1314-1321. 